\title{
BMJ Open Clinical examination, critical care ultrasonography and outcomes in the critically ill: cohort profile of the Simple Intensive Care Studies-I
}

\author{
Bart Hiemstra, ${ }^{1}$ Ruben J Eck, ${ }^{1}$ Geert Koster, ${ }^{1}$ Jørn Wetterslev, ${ }^{2}$ Anders Perner, ${ }^{3}$ \\ Ville Pettilä, ${ }^{4}$ Harold Snieder, ${ }^{5}$ Yoran M Hummel, ${ }^{6}$ Renske Wiersema, ${ }^{1}$ \\ Anne Marie G A de Smet, ${ }^{1}$ Frederik Keus, ${ }^{1}$ Iwan C C van der Horst, ${ }^{1}$ SICS Study \\ Group
}

To cite: Hiemstra B, Eck RJ, Koster G, et al. Clinical examination, critical care ultrasonography and outcomes in the critically ill: cohort profile of the Simple Intensive Care Studies-I. BMJ Open 2017;7:e017170. doi:10.1136/ bmjopen-2017-017170

- An additional supplementary table is available online

- Prepublication history and additional material are available. To view these files please visit the journal online (http://dx.doi org/10.1136/bmjopen-2017017170).

$\mathrm{BH}$ and RJE contributed equally.

Received 5 April 2017 Revised 22 May 2017 Accepted 7 June 2017

CrossMark

For numbered affiliations see end of article.

Correspondence to

Bart Hiemstra;

b.hiemstra01@umcg.nl

\section{ABSTRACT}

Purpose In the Simple Intensive Care Studies-I (SICS-I), we aim to unravel the value of clinical and haemodynamic variables obtained by physical examination and critical care ultrasound (CCUS) that currently guide daily practice in critically ill patients. We intend to (1) measure all available clinical and haemodynamic variables, (2) train novices in obtaining values for advanced variables based on CCUS in the intensive care unit (ICU) and (3) create an infrastructure for a registry with the flexibility of temporarily incorporating specific (haemodynamic) research questions and variables. The overall purpose is to investigate the diagnostic and prognostic value of clinical and haemodynamic variables.

Participants The SICS-I includes all patients acutely admitted to the ICU of a tertiary teaching hospital in the Netherlands with an ICU stay expected to last beyond 24 hours. Inclusion started on 27 March 2015.

Findings to date On 31 December 2016, 791 eligible patients fulfilled our inclusion criteria of whom 704 were included. So far 11 substudies with additional variables have been designed, of which six were feasible to implement in the basic study, and two are planned and awaiting initiation. All researchers received focused training for obtaining specific CCUS images. An independent Core laboratory judged that 632 patients had CCUS images of sufficient quality.

Future plans We intend to optimise the set of variables for assessment of the haemodynamic status of the critically ill patient used for guiding diagnostics, prognosis and interventions. Repeated evaluations of these sets of variables are needed for continuous improvement of the diagnostic and prognostic models. Future plans include: (1) more advanced imaging; (2) repeated clinical and haemodynamic measurements; (3) expansion of the registry to other departments or centres; and (4) exploring possibilities of integration of a randomised clinical trial superimposed on the registry.

Study registration number NCT02912624; Pre-results.

\section{INTRODUCTION}

Circulatory shock occurs in about one-third of all critically ill patients. ${ }^{1}$ These patients

\section{Strengths and limitations of this study}

- The large prospective design is powered to evaluate the value of combinations of clinical and haemodynamic variables for guiding decisions in acutely admitted critically ill patients.

- The flexible simple design allows expansions in time and/or place and/or other research questions.

- The basic study is limited to a one-time evaluation of variables.

- Critical care ultrasound measurements are not obtainable in all critically ill patients due to positioning issues or insufficient image quality.

have an increased risk of multiorgan failure, long-term morbidity and mortality. ${ }^{2}{ }^{3} \mathrm{~A}$ recent consensus statement on circulatory shock recommends to use a combination of clinical, biochemical and/or haemodynamic variables, varying from simple to advanced, for establishing the diagnosis and instigation of treatment. ${ }^{4}$ The consensus advocates frequent measurement of heart rate, blood pressure, body temperature, skin perfusion, urine output and mental status. ${ }^{4}$ If necessary, more advanced and sequential haemodynamic assessments using critical care ultrasound (CCUS) as preferred modality are recommended. ${ }^{4-7}$ Previous studies have found different prognostic or diagnostic variables, many have presented single or dual variable associations and no research has evaluated their additional value on top of the accepted predictors. Different studies highlight different predictors of mortality: low blood pressures, ${ }^{8-11}$ increased lactate levels, ${ }^{8} 9^{11-14}$ prolonged capillary refill times,${ }^{15-17}$ skin mottling, ${ }^{18-20}$ oliguria $^{2122}$ and decreased cardiac output ${ }^{23} 24$ are identified as prognostic variables. These contrasting 
results are also seen in studies that investigated the value of clinical and biochemical variables for diagnosing shock. ${ }^{17182325-34}$ The reason for inconsistency of results in these studies potentially originate from several methodological flaws, including improper research design, lack of confirmation cohorts and power and sample size issues. Several studies selected specific subpopulations (eg, patients with sepsis, ${ }^{8-11} 13171833$ acute cardiac failure $^{1623}$ or trauma ${ }^{15}$ ) and some had a retrospective design or used convenience samples from large databases. ${ }^{9-12} 152021$ Most studies analysed single variable associations rather than evaluating their additional predictive value on top of the widely accepted variables using multivariate models. $^{8} 17182123$ Also, most had relatively small sample sizes. ${ }^{8-10} 12$ 15-18 232433

Both clinical and haemodynamic variables used for diagnosing shock are currently recommended as 'best practice'. ${ }^{4}$ Both the consensus and the Surviving Sepsis Campaign guideline recommend further haemodynamic assessment (such as cardiac function) to determine the type of shock if the clinical examination does not lead to a clear diagnosis (best practice statement). ${ }^{45}$ Less invasive devices are recommended instead of more invasive devices only when they have been validated in the context of patients with shock. ${ }^{436}$

The additive diagnostic and prognostic value of combinations of clinical, biochemical and haemodynamic variables remains to be established with a higher quality of evidence. These variables have never been evaluated collectively in a large, unselected, prospective cohort of critically ill patients. Therefore, we established the Simple Intensive Care Studies-I (SICS-I) with the aim to evaluate the diagnostic and prognostic value of a comprehensive selection of clinical and haemodynamic variables in the critically ill. This paper describes the study protocol with the diagnostic and prognostic aims of the basic study as well as the characteristics of the patients included in the cohort so far. All substudies will be presented here for illustrative purposes without elaborating on each specific rationale and design.

\section{COHORT DESCRIPTION \\ Participants}

This prospective cohort study is conducted in the Department of Critical Care of the University Medical Center Groningen (UMCG), a tertiary teaching hospital in the Northern part of the Netherlands. Our intensive care unit (ICU) has 44 beds divided over four subunits to which all types of critically ill adult patients are admitted. In our department, CCUS is available if considered indicated to inform practice but is not performed in each patient each day. We initiated our study in one unit to deal with start-up issues and to assess feasibility, including (1) whether it was logistically possible to include a broad population of acutely admitted critically ill patients, (2) whether all clinical and haemodynamic measurements could be recorded within a limited time so that it did not obstruct routine patient care and (3) whether novices could obtain CCUS images of sufficient quality after training. The entire study was purely observational in design; no interventions were applied. After inclusion of our first patient on 27 March 2015, we gradually expanded inclusions to all the four subunits of the department within 1 year.

All acutely admitted adult critically ill patients expected to stay beyond 24 hours were included on their first day of ICU admission. The attending intensivist estimated the expected duration of ICU treatment. Inclusions and measurements were obtained by medical research interns and PhD students who were not in any way involved in patient care. All measurements, including CCUS findings, were not revealed to the care providers. If any possible abnormality was observed by CCUS, an independent qualified intensivist was contacted for judgement of informing the attending intensivist. All researchers underwent a focused CCUS training by experienced cardiologist-intensivists. Following hospital regulations, patients or their legal representatives were informed and were excluded if they refused to participate. Other reasons for exclusion were acute psychiatric disorders, mental retardation, serious language barriers, continuous resuscitation efforts or mechanical circulatory support. The local institutional review board (Medisch Ethische Toetsingscommissie (METc) of the UMCG; M15.168207) approved the main study and the additional measures added when initiating the substudies (METc M11.104639 and M16.193856).

\section{Registry, substudies and research questions}

The cohort study was designed to register a set of baseline clinical, biochemical and haemodynamic variables in each included patient. In the initial basic study, we collected baseline demographic data, clinical data by protocolised physical examination and CCUS data to measure cardiac output (see online supplementary file 1). The co-primary aims are to determine the association between variables measured by physical examination and cardiac output and to assess the prognostic value of both the clinical and haemodynamic variables for 90-day mortality. The flexible design in terms of research questions, planning and data collection allows incorporation of substudies with additional measurements to assess the feasibility in terms of the quality of the measurements (figure 1). The designed substudies with their specific research questions and (temporarily) added variables are listed in table 1. After completion of SICS-I, we will analyse the variables to establish an optimised basic set of variables as SICS-II (figure 1).

\section{Data collection}

On inclusion, we collected a clinical and haemodynamic profile of each patient by physical examination, recording data from the bedside monitor as well as using CCUS. All variables and the measurements were predefined in a protocol (see online supplementary file 1). In the current study, CCUS comprised transthoracic 

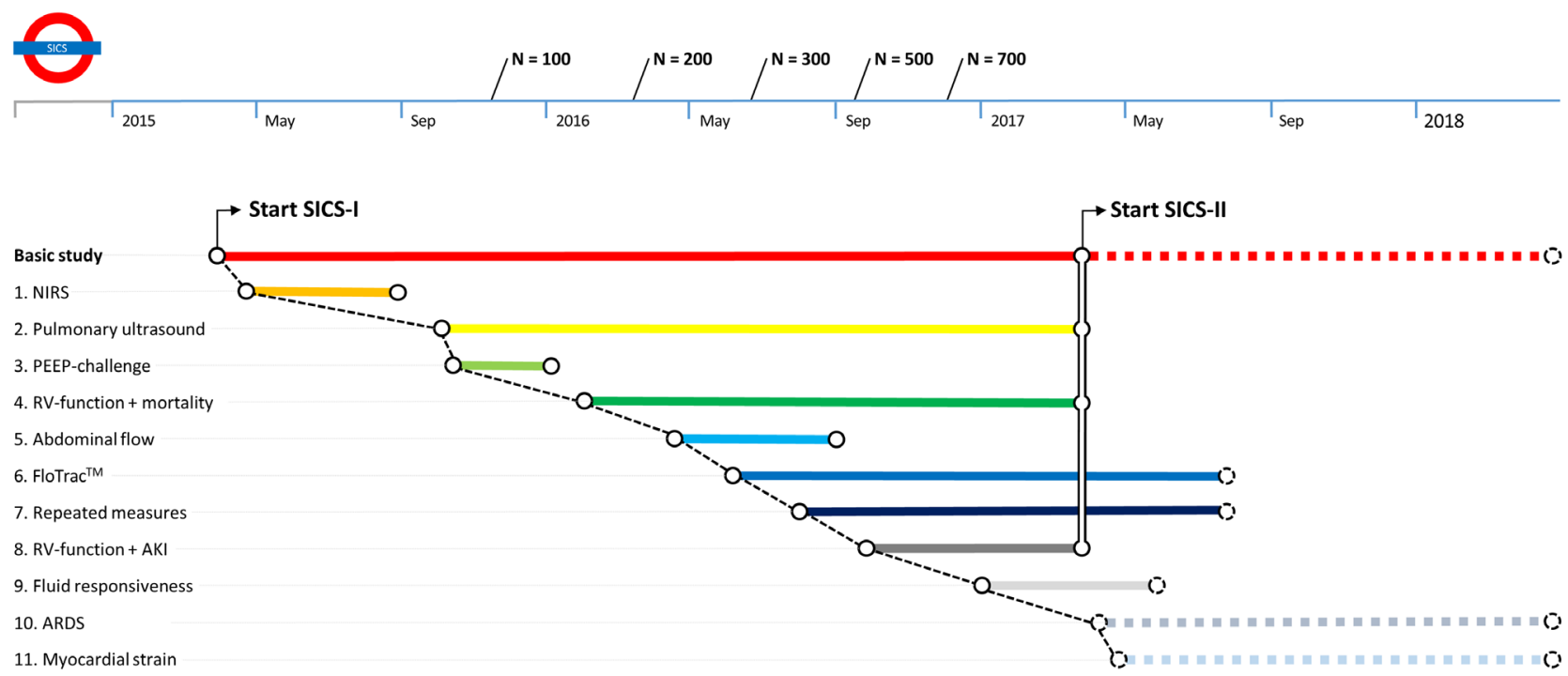

Figure 1 Timeline of basic study line and substudies. The substudies are explained in table 1. AKI, acute kidney injury; ARDS, acute respiratory distress syndrome; NIRS, near-infrared spectroscopy; PEEP, positive end-expiratory pressure; RV, right ventricular; SICS, Simple Intensive Care Studies.

echocardiography (basic study, substudies 1, 3-9 and 11), pulmonary ultrasound (substudy 3 and 10) and ultrasonography of the large arteries (substudy 5). The CCUS images and measurements were validated by an independent core laboratory (Groningen Image Core Lab, UMCG, Groningen, the Netherlands, www.gicl.com). These echo laboratory technicians were blinded for all other measurements. Other variables such as laboratory values and diagnoses as judged by an ICU physician at ICU admission and discharge were obtained from the patient's electronic medical records. Comorbidities, relevant medical history and Acute Physiology and Chronic Health Evaluation II and IV (APACHE II and IV) scores were registered as well. All-cause mortality at 90-day follow-up was based on the municipal personal records database.

\section{Data management}

The protocol of this study and its substudies was published on the intranet of our hospital before the start of the study (project number: 201500144) and, after completion of the pilot phase, was also registered at clinicaltrials. gov (NCT02912624). A customised electronic case report form (e-CRF) was developed prior to study onset in OpenClinica version 3.9 (OpenClinica, LLC and collaborators, Waltham, Massachusetts, USA). Patient data were pseudoanonymised and entered in OpenClinica, and a decoding list of included patients was kept separate from the e-CRF by the research office of the Department of
Critical Care. All measurements, informed consent forms and other patient details were uploaded in OpenClinica and stored on a secure hospital server. Independent study monitoring of the organisation and the conduct of the study was in adherence to the Good Clinical Practice guidelines. $^{37}$

\section{FINDINGS TO DATE}

\section{Characteristics of study population}

Between 21 March 2015 and 31 December 2016, all eligible patients were identified by daily screening of all new patients admitted to the ICU (figure 2). Currently, 704 patients have been included from a total of 791 eligible patients. The mean age was $61( \pm 15)$ years and 457 patients $(65 \%, 95 \%$ CI $61 \%$ to $68 \%)$ were male. Table 2 displays demographic data along with all haemodynamic variables of the basic study. There were no missing data for the following variables: blood pressures, heart rate, urine output, central temperature, arterial haemoglobin and lactate levels. Mottling scores, capillary refill times and peripheral temperatures had missing values in $2 \%-8 \%$ of the patients.

Reasons for missing data included a dark or icteric skin colour (mottling and capillary refill times) and compression stockings (capillary refill time at the knee and peripheral temperature at the dorsum of the foot). 
Table 1 Specific research questions with add-on measurements ${ }^{53}$

Short title Research questions

Basic study Which combination of clinical variables obtainable through physical examination is associated with cardiac output measured by critical care ultrasonography (CCUS) ${ }^{53}$ ? Which combination of clinical and haemodynamic variables is associated with 7-day, 30-day and 90 -day mortality?

1. NIRS What is the association of clinical and haemodynamic variables and tissue (muscle) oxygen saturation $\left(\mathrm{StO}_{2}\right)$ measured by near-infrared spectroscopy (NIRS)?

Does the kneecap NIRS measurement have a better association with the clinical and haemodynamic variables than the NIRS measurement at the thenar muscle?

2. Pulmonary ultrasound What is the association of a B-profile* measured with pulmonary ultrasonography and auscultation for pulmonary crackles with the diagnosis of pulmonary oedema by chest radiograph?

Is there a difference in cardiac output between the group with and without the presence of a B-profile?

3. PEEP-challenge Does an increase in positive end-expiratory pressure (PEEP) correlate with a decrease in cardiac output?

4. RV-function + mortality What is the association between right ventricular (RV)-function assessed with tricuspid annular plane systolic excursion and peak tissue Doppler systolic velocity in the tricuspid annulus (RV S') with 90-day mortality?

What is the association between RV-function and clinical variables obtained from through physical examination?

5. Abdominal flow Is there a correlation between cardiac output and peripheral blood flow measured with CCUS? Can we calculate a proxy for abdominal organ blood flow by subtraction of peripheral flow to head and extremities from the cardiac output?

6. FloTrac $\dagger \quad$ What is the level of agreement between cardiac output measured by the FloTrac compared with cardiac output measured with CCUS?

Do the levels of agreement change when factors that might influence FloTrac measurements are present?

7. Repeated measures What is the association of clinical variables with the cardiac output measured on two different time-points: one within the first 24 hours of admission and a second 24 hours thereafter

8. RV-function + AKI Is RV volume overload measured by tricuspid insufficiency and RV diameter associated with acute kidney injury (AKI)?

9. Fluid responsiveness Do variations in end-tidal carbon dioxide $\left(\mathrm{EtCO}_{2}\right)$, heart rate and blood pressure induced by the passive leg raising (PLR) test predict fluid responsiveness?

Does a PLR test without lowering the head of the bed have a similar accuracy compared with the standard PLR test?

Will a temporary increase of PEEP lead to a greater decrease in cardiac output in fluid responders compared with non-responders?

10. ARDS

What is the association between CCUS measurements and the presence or development of acute respiratory distress syndrome (ARDS) during the first 24 hours of ICU stay?

11. Myocardial strain Is left ventricular and RV myocardial strain measured with tissue Doppler imaging a predictor of 90-day mortality?

What is the association betweenmyocardial strain measured with tissue Doppler imaging and conventional CCUS measurements?

${ }^{*} \mathrm{~B}$-profile: A B-profile is a strong indicator of pulmonary oedema and is present when three or more B lines are seen in at least three of the six BLUE points, or in two of the four lower BLUE points. ${ }^{54}$

†FloTrac: the FloTrac (Edwards Lifesciences, Irvine, California, USA) is a pulse contour technique that analyses the arterial pressure waveform to compute stroke volume and cardiac output. The technique consists a dedicated pressure sensor (FloTrac) and a monitor to compute stroke volume and cardiac output (Vigileo). ${ }^{55}$

Vasopressors and/or inotropes were used in 363 patients $(52 \%, 95 \%$ CI $48 \%$ to $55 \%)$, of whom 351 patients $(50 \%$, $95 \%$ CI $46 \%$ to $54 \%)$ were given noradrenalin, $10(1 \%$, $95 \%$ CI $1 \%$ to $3 \%)$ vasopressin, $29(4 \%, 95 \%$ CI $3 \%$ to $6 \%)$ milrinone and 20 (3\%, 95\% CI $2 \%$ to $4 \%)$ dobutamine. In 46 patients (7\%, $95 \%$ CI $5 \%$ to $9 \%$ ), more than one type of vasopressor or inotrope was administered. The median APACHE IV score of our population was 74 (IQR 57-92) with a corresponding associated risk of in-hospital mortality of $25 \%{ }^{38}$ At 90-day follow-up, 173 patients $(25 \%, 95 \%$ CI $22 \%$ to $28 \%)$ had died, and 6 patients $(1 \%$, $95 \%$ CI $0 \%$ to $2 \%$ ) were lost to follow-up due to emigration or residence in another country. 
3892 Assessed for eligibility

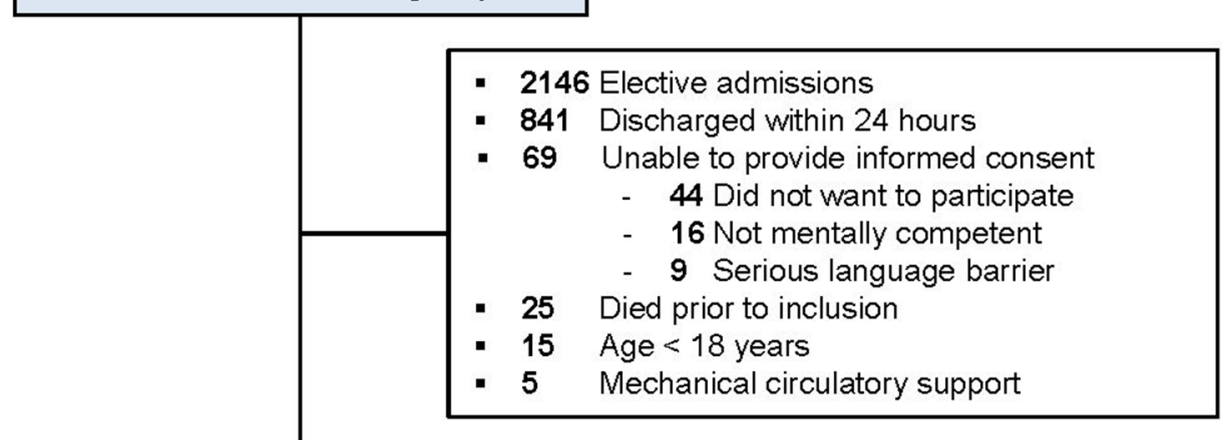

791 Fulfilled inclusion criteria

87 Excluded due to one of the following reasons:

- 68 No echocardiography possible

- 19 Logistic reasons

704 Included in SICS-I cohort

72 Echocardiography images of insufficient quality

632 Included in final analyses

Figure 2 Flow diagram of the Simple Intensive Care Studies-I (SICS-I) cohort. CCUS, critical care ultrasonography

\section{Critical care ultrasound}

Cardiac output measurements by CCUS were performed in all the 704 included patients. After validation by the independent core laboratory $632(90 \%, 95 \%$ CI $87 \%$ to $92 \%$ ) CCUS images were judged to be of sufficient quality for reliable cardiac output measurement. CCUS could not be performed in 68 patients due to various reasons obstructing the CCUS window, such as thoracic drains, postsurgical incisions, wounds or (subcutaneous) emphysema (figure 2).

\section{Substudies}

To date, we have initiated nine substudies that focus on more clinical or haemodynamic measurements (table 3). After evaluation of finished substudies (substudies 1-7), we considered 'pulmonary ultrasound for the presence or absence of B-lines' (substudy 2), 'the right ventricle function' (substudy 4), 'validation of the FloTrac cardiac output measurement device' (substudy 6) and 'serial clinical and haemodynamic measurements' (substudy 7) to be feasible. These substudies were successively implemented in the basic study line and data collection is still ongoing. Substudies 1, 3 and 5 were discontinued as results appeared inaccurate or were too time-consuming. Currently, two substudies are running (8 and 9 ), and two new substudies are planned (10 and 11) (figure 1).

\section{Future perspectives}

In the near future, we aim to expand our SICS studies to patients in the emergency room, independent of their destination ward in the hospital. We expect that both the diagnostic and prognostic value of available clinical and haemodynamic variables will change when patients are included in earlier phase of acute illness. Other future possibilities may include even more advanced CCUS imaging, other specific haemodynamic research questions and expansion to other national and/or international centres. Ultimately a randomised clinical trial for evaluation of haemodynamic interventions may be superimposed on this registry with the ultimate goal to improve patient-centred outcomes. $^{39}$

\section{STRENGTHS AND LIMITATIONS}

We have described the study protocol including the research questions and design as well as the characteristics of the patients included in the cohort so far. The strengths of the study include the prospective design allowing systematic data collection following a predefined protocol. All variables are measured according to strict definitions in a broad ICU population that represents the daily critical care in a university hospital. We already learnt that values of clinical and haemodynamic variables show large individual differences in the critically ill patients of the SICS-I cohort. Currently in critical care all patients are assessed by the same combination of clinical and haemodynamic variables. It is very likely that these variables will have different diagnostic and prognostic value depending on patient subgroups. A more personalised approach for clinical and haemodynamic assessment in 
Table 2 Clinical, haemodynamic and biochemical variables of the basic study

\begin{tabular}{|c|c|}
\hline Variable & Total population $(n=704)$ \\
\hline \multicolumn{2}{|l|}{ Clinical variables } \\
\hline Age, years* & $61 \pm 15$ \\
\hline Body mass index, $\mathrm{kg} / \mathrm{m}^{2 *}$ & $26.83 \pm 5.87$ \\
\hline Time to inclusion, hours $†$ & $14.9(8.2,19.8)$ \\
\hline Positive end-expiratory pressure, $\mathrm{cmH}_{2} \mathrm{O}^{\dagger}$ & $7(5,8)$ \\
\hline Heart rate, beats per $\min ^{*}$ & $89 \pm 21$ \\
\hline Atrial fibrillation, $\mathrm{n}(\%)$ & $47(7 \%, 95 \% \mathrm{Cl} 5 \%$ to $9 \%)$ \\
\hline Systolic blood pressure, mm Hg† & $114(100,132)$ \\
\hline Diastolic blood pressure, $\mathrm{mm} \mathrm{Hg} \dagger$ & $58(52,65)$ \\
\hline Urine output over 1 hour, mL/kg/h† & $0.52(0.26,1.07)$ \\
\hline Urine output over 6 hours, $\mathrm{mL} / \mathrm{kg} / \mathrm{h} \dagger$ & $0.56(0.32,1.06)$ \\
\hline Central temperature, ${ }^{\circ} \mathrm{C}^{\star}$ & $37.0 \pm 0.9$ \\
\hline Peripheral temperature, ${ }^{\circ} \mathrm{C}^{\star}$ & $28.1 \pm 4.0$ \\
\hline Central-to-peripheral delta temperature, ${ }^{\circ} \mathrm{C}^{*}$ & $9.0 \pm 3.9$ \\
\hline Cold extremities, subjective, $\mathrm{n}(\%)$ & $276(39 \%, 95 \% \mathrm{Cl} 35 \%$ to $43 \%)$ \\
\hline Capillary refill time sternum, s† & $3.0(2.0,3.0)$ \\
\hline Capillary refill time finger, s† & $2.5(2.0,4.0)$ \\
\hline Capillary refill time knee, s† & $3.0(2.0,5.0)$ \\
\hline Cardiac index, $\mathrm{L} / \mathrm{min} / \mathrm{m}^{2 *}$ & $2.63 \pm 0.99$ \\
\hline
\end{tabular}

\section{Biochemical values}

\begin{tabular}{ll} 
Haemoglobin, $\mathrm{mmol} / \mathrm{L}^{*}$ & $6.7 \pm 1.5$ \\
Lactate, $\mathrm{mmol} / \mathrm{L} \dagger$ & $1.5(0.9,2.3)$ \\
\hline
\end{tabular}

${ }^{*}$ Mean \pm SD.

†Median (IQR).

this heterogeneous population seems to be appropriate. We therefore aim to include a sufficient number of critically ill patients to establish the additional diagnostic and prognostic value of specific clinical and haemodynamic variables in predefined subgroups, for example, patients with sepsis, shock or acute respiratory distress syndrome.

Another strength is the flexible design that allows temporary or definite incorporation of specific haemodynamic research questions. This design facilitates a combined evaluation of clinical and haemodynamic variables used in daily practice and offers the possibility of evaluating whether new and/or advanced measurements can improve diagnostic and prognostic accuracy.
Furthermore, evaluations of substudies adapt the set of clinical and haemodynamic variables to be measured in the basic study line. Evaluations of inclusions or exclusions of variables are based on the additional diagnostic and prognostic value as well as the efforts and possible interference with patient care needed for recording.

A lesson learnt from this cohort study is that novices, that is, senior medical and $\mathrm{PhD}$ students, can be effectively trained to obtain advanced haemodynamic variables derived from CCUS. While previous studies showed that non-cardiological professionals could obtain reliable CCUS images and measurements after an education programme, ${ }^{40-42}$ we demonstrated that this possibility 
Table 3 Clinical or haemodynamic variables measured in substudies

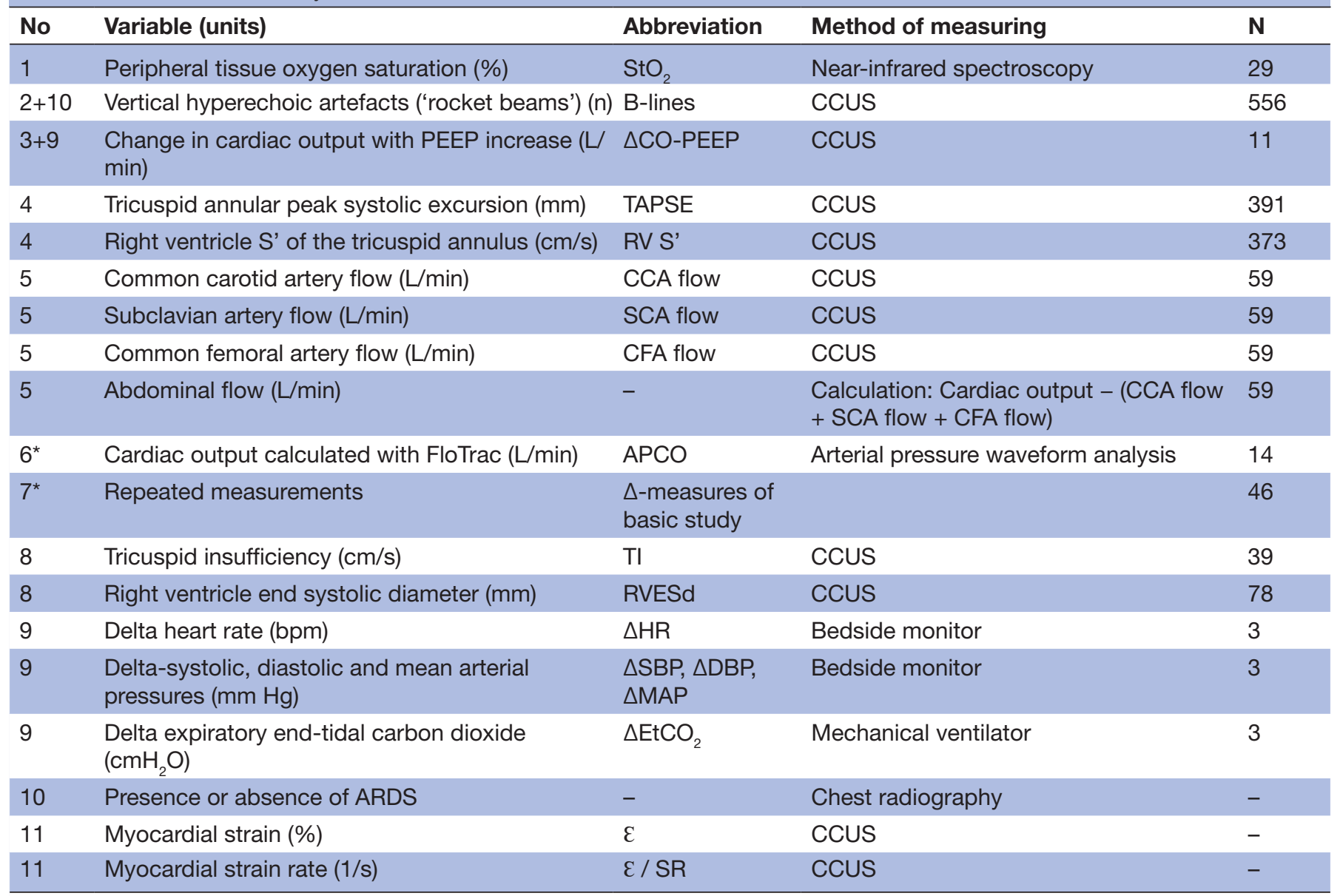

${ }^{*}$ Sub-studies 6 and 7 include only patients in a state of circulatory shock.

ARDS, acute respiratory distress syndrome; CCUS, critical care ultrasound; N,number; PEEP, positive end-expiratory pressure.

seems to apply to novices as well. Recently, CCUS is increasingly applied in different areas of medicine including the prehospital phase, the emergency department and the ICU setting. ${ }^{42-46}$ CCUS is highly operator dependent and time consuming. Due to validation of all our measurements by an echographic core laboratory, we ensured the quality of our echographic images and accuracy of the related measurements. Despite training and independent validation of our measurements, the quality and accuracy of our images may still be inferior compared with those obtained by skilled echolaboratory technicians, cardiologists, intensivists or cardiologist-intensivists. However, our researchers scheduled themselves 7 days a week and can easily expand to other potential study locations. A limitation inherent to the technique is that CCUS cannot be performed in patients with pathology interfering with a proper ultrasound window view, such as drains, wounds and subcutaneous emphysema. This limitation will, however, apply to both inexperienced and experienced investigators.

One limitation for statistical interference of the current study design is that we will encounter multiplicity issues due to multiple testing of the data across substudies. Repeated testing may result in increased type I errors.
Additionally, all substudies need separate detailed sample size considerations. As a rule of thumb, at least 10 events are necessary for each variable included in a final model. ${ }^{47}$ To account for this potential multiplicity issue combined with multiple sample size considerations, we emphasise the hypothesis-generating aspect of results and advocate that findings should be validated in an independent cohort.

Another major limitation of our SICS-I basic study is that all measurements are limited to a single time point. To expect, for example, that one single cardiac output measurement may predict mortality is obviously unrealistic as cardiac output may vary widely over time. ${ }^{49} 50$ One of our substudies specifically aims to determine the diagnostic accuracy of continuously monitoring cardiac output in patients with shock. Other CCUS measurements with less variation over time might eventually appear to be better predictors, and we are currently exploring which set of CCUS measurements may accommodate clinical needs. However, in daily practice, snapshot measurements guide treatment decisions as triggers for interventions. Ideally, decisions for interventions will be informed by (trends of) repeated or even continuous measurements of both cardiac output and other 
haemodynamic variables. Continuous measurement is limited to the variables heart rate, blood pressures and non-invasively measured cardiac output. The use of either invasive or non-invasive continuous monitoring of cardiac output has no documented benefit on mortality ${ }^{51}$ which might result from either lack of prognostic additive value, insufficient diagnostic accuracy, the treatment algorithm used or absence of any beneficial effects of the selected treatment interventions. ${ }^{52}$ The entire process of setting the correct diagnosis, implementing an appropriate intervention, monitoring treatment effect and eventually improving patient prognosis in a haemodynamically unstable patient is an extremely complex chain of events. Evidence-based evaluation of such a process with complex time-dependent repeated interactions between diagnosis and treatment requires an approach that includes all three types of research: diagnostic, prognostic and a combination of intervention with prognostic research. For the latter, our study could serve as an infrastructure to conduct randomised clinical trials.

\section{COLLABORATION}

We have described the study protocol and the characteristics of the patients. The aim of the SICS-I study is to serve as a pilot for a large, multicentre, preferably multinational registry aimed at evaluating simple clinical and haemodynamic measures to guide treatment decisions focused on haemodynamics. Our experience with the SICS-I will fuel future projects and the selection of clinical and haemodynamic variables in large-scale collaborations. This experience has already led to an ongoing collaboration with the Copenhagen Trial Unit, the Centre for Research in Intensive Care and The Division of Intensive Care Medicine of the Helsinki University Hospital, and we are open for any further suggestions or proposals for collaboration.

\section{Author affiliations}

${ }^{1}$ Department of Critical Care, University of Groningen, University Medical Center Groningen, Groningen, The Netherlands

${ }^{2}$ The Copenhagen Trial Unit, Centre for Clinical Intervention Research, Rigshospitalet, Copenhagen University Hospital, Copenhagen, Denmark ${ }^{3}$ Department of Intensive Care, Centre for Research in Intensive Care, Rigshospitalet, Copenhagen University Hospital, Copenhagen, Denmark ${ }^{4}$ Division of Intensive Care Medicine, Department of Anesthesiology, Intensive Care and Pain Medicine, University of Helsinki and Helsinki University Hospital, Helsinki, Finland

${ }^{5}$ Department of Epidemiology, University of Groningen, University Medical Center Groningen, Groningen, The Netherlands

${ }^{6}$ Department of Cardiology, University of Groningen, University Medical Center Groningen, Groningen, The Netherlands

Collaborators R.P. Clement, W. Dieperink, P. van der Harst, D.H. Hilbink, M. Klasen, M. Klaver, T. Kaufmann, T.W.L. Scheeren, L.J. Schokking, V.W. Sikkens.

Contributors IvdH and FK created the idea of and supervised the SICS-I study. $\mathrm{BH}, \mathrm{RJE}, \mathrm{GK}$ and RW developed the protocol, created the educational platform and implemented the study. BH and RJE drafted the initial versions of the manuscript, analysed the data and contributed equally to this manuscript. JW, AP, VP and HS were involved in future expansions within an international consortium. All other authors critically reviewed the manuscript and agreed with the final version and findings.
Funding This research received no specific grant from any funding agency in the public, commercial or not-for-profit sectors.

Competing interests None declared.

Patient consent The manuscript does not contain any identifiable medical information. For our informed consent procedure, we refer to our manuscript.

Ethics approval Medisch Ethische Toetsingscommissie, University Medical Center Groningen.

Provenance and peer review Not commissioned; externally peer reviewed.

Data sharing statement Upon completion of our study, anonymised data will be available on reasonable request.

Open Access This is an Open Access article distributed in accordance with the Creative Commons Attribution Non Commercial (CC BY-NC 4.0) license, which permits others to distribute, remix, adapt, build upon this work non-commercially, and license their derivative works on different terms, provided the original work is properly cited and the use is non-commercial. See: http://creativecommons.org/ licenses/by-nc/4.0/

(c) Article author(s) (or their employer(s) unless otherwise stated in the text of the article) 2017. All rights reserved. No commercial use is permitted unless otherwise expressly granted.

\section{REFERENCES}

1. Sakr Y, Reinhart K, Vincent JL, et al. Does dopamine administration in shock influence outcome? Results of the Sepsis Occurrence in Acutely III Patients (SOAP) Study. Crit Care Med 2006;34:589-97.

2. De Backer D, Biston P, Devriendt J, et al. Comparison of dopamine and norepinephrine in the treatment of shock. N Engl J Med 2010;362:779-89.

3. Vincent JL, Marshall JC, Namendys-Silva SA, et al. Assessment of the worldwide burden of critical illness: the intensive care over nations (ICON) audit. Lancet Respir Med 2014;2:380-6.

4. Cecconi M, De Backer D, Antonelli M, et al. Consensus on circulatory shock and hemodynamic monitoring. Task force of the European Society of Intensive Care Medicine. Intensive Care Med 2014:40:1795-815.

5. Cholley BP, Vieillard-Baron A, Mebazaa A. Echocardiography in the ICU: time for widespread use!. Intensive Care Med 2006;32:9-10.

6. Salem R, Vallee F, Rusca M, et al. Hemodynamic monitoring by echocardiography in the ICU: the role of the new echo techniques. Curr Opin Crit Care 2008;14:561-8.

7. Vincent JL, De Backer D. Circulatory shock. N Engl J Med 2013;369:1726-34.

8. Bernardin G, Pradier C, Tiger F, et al. Blood pressure and arterial lactate level are early indicators of short-term survival in human septic shock. Intensive Care Med 1996;22:17-25.

9. Varpula M, Tallgren M, Saukkonen K, et al. Hemodynamic variables related to outcome in septic shock. Intensive Care Med 2005;31:1066-71.

10. Dünser MW, Takala J, Ulmer H, et al. Arterial blood pressure during early sepsis and outcome. Intensive Care Med 2009;35:1225-33.

11. Houwink AP, Rijkenberg S, Bosman RJ, et al. The association between lactate, mean arterial pressure, central venous oxygen saturation and peripheral temperature and mortality in severe sepsis: a retrospective cohort analysis. Crit Care 2016;20:1243-3.

12. Tuchschmidt J, Fried J, Swinney R, et al. Early hemodynamic correlates of survival in patients with septic shock. Crit Care Med 1989;17:719-23.

13. De Backer D, Donadello K, Sakr Y, et al. Microcirculatory alterations in patients with severe Sepsis: impact of time of assessment and relationship with outcome. Crit Care Med 2013;41:791-9.

14. Varis E, Pettilä V, Poukkanen M, et al. Evolution of blood lactate and 90-Day mortality in septic shock. A Post Hoc analysis of the FINNAKI Study. Shock 2017;47:574-81.

15. Champion HR, Sacco WJ, Hannan DS, et al. Assessment of injury severity: the triage index. Crit Care Med 1980;8:201-8.

16. van Genderen ME, Lima A, Akkerhuis M, et al. Persistent peripheral and microcirculatory perfusion alterations after out-of-hospital cardiac arrest are associated with poor survival. Crit Care Med 2012;40:2287-94.

17. Ait-Oufella $\mathrm{H}$, Bige $\mathrm{N}$, Boelle $\mathrm{PY}$, et al. Capillary refill time exploration during septic shock. Intensive Care Med 2014;40:958-64.

18. Ait-Oufella $\mathrm{H}$, Lemoinne $\mathrm{S}$, Boelle $\mathrm{PY}$, et al. Mottling score predicts survival in septic shock. Intensive Care Med 2011;37:801-7. 
19. Coudroy R, Jamet A, Frat JP, et al. Incidence and impact of skin mottling over the knee and its duration on outcome in critically ill patients. Intensive Care Med 2015;41:452-9.

20. de Moura EB, Amorim FF, da Cruz Santana AN, et al. Skin mottling score as a predictor of 28-day mortality in patients with septic shock. Intensive Care Med 2016;42:479-80.

21. Macedo E, Malhotra R, Bouchard J, et al. Oliguria is an early predictor of higher mortality in critically ill patients. Kidney Int 2011;80:760-7.

22. Vaara ST, Parviainen I, Pettilä V, et al. Association of oliguria with the development of acute kidney injury in the critically ill. Kidney Int 2016;89:200-8.

23. Forrester JS, Diamond GA, Swan HJ. Correlative classification of clinical and hemodynamic function after acute myocardial infarction. Am J Cardiol 1977;39:137-45.

24. Parker MM, Shelhamer JH, Natanson C, et al. Serial cardiovascular variables in survivors and nonsurvivors of human septic shock: heart rate as an early predictor of prognosis. Crit Care Med 1987;15:923-9.

25. Joly HR, Weil MH. Temperature of the great toe as an indication of the severity of shock. Circulation 1969;39:131-8.

26. Woods I, Wilkins RG, Edwards JD, et al. Danger of using core/ peripheral temperature gradient as a guide to therapy in shock. Crit Care Med 1987;15:850-2.

27. Tuchschmidt J, Sharma OP. Impact of hemodynamic monitoring in a medical intensive care unit. Crit Care Med 1987;15:840-3.

28. Vincent JL, Moraine JJ, van der Linden P. Toe temperature versus transcutaneous oxygen tension monitoring during acute circulatory failure. Intensive Care Med 1988;14:64-8.

29. Connors AF, Dawson NV, Shaw PK, et al. Hemodynamic status in critically ill patients with and without acute heart disease. Chest 1990;98:1200-6.

30. Wo CC, Shoemaker WC, Appel PL, et al. Unreliability of blood pressure and heart rate to evaluate cardiac output in emergency resuscitation and critical illness. Crit Care Med 1993;21:218-23.

31. Kaplan LJ, McPartland K, Santora TA, et al. Start with a subjective assessment of skin temperature to identify hypoperfusion in intensive care unit patients. J Trauma 2001;50:620-8.

32. Boerma EC, Kuiper MA, Kingma WP, et al. Disparity between skin perfusion and sublingual microcirculatory alterations in severe Sepsis and septic shock: a prospective observational study. Intensive Care Med 2008;34:1294-8.

33. Bourcier S, Pichereau C, Boelle PY, et al. Toe-to-room temperature gradient correlates with tissue perfusion and predicts outcome in selected critically ill patients with severe infections. Ann Intensive Care 2016;6:016-164.

34. Hiemstra B, Eck RJ, Keus F, et al. Clinical examination for diagnosing circulatory shock. Curr Opin Crit Care 2017;23:293-301.

35. Rhodes A, Evans LE, Alhazzani W, et al. Surviving sepsis campaign: international guidelines for management of sepsis and septic shock: 2016. Intensive Care Med 2017;43:304-77.

36. Teboul JL, Saugel B, Cecconi M, et al. Less invasive hemodynamic monitoring in critically ill patients. Intensive Care Med 2016;42:1350-9.

37. Anonymous JL. ICH harmonised tripartite guideline: guideline for good clinical practice. J Postgrad Med 2001;47:199-203.
38. Zimmerman JE, Kramer AA, McNair DS, et al. Acute Physiology and Chronic Health Evaluation (APACHE) IV: hospital mortality assessment for today's critically ill patients. Crit Care Med 2006;34:1297-310.

39. Guyatt GH, Oxman AD, Kunz R, et al. What is "quality of evidence" and why is it important to clinicians? BMJ 2008;336:995-8.

40. Beraud AS, Rizk NW, Pearl RG, et al. Focused transthoracic echocardiography during critical care medicine training: curriculum implementation and evaluation of proficiency*. Crit Care Med 2013;41:e179-81.

41. Díaz-Gómez JL, Perez-Protto S, Hargrave J, et al. Impact of a focused transthoracic echocardiography training course for rescue applications among anesthesiology and critical care medicine practitioners: a prospective study. J Cardiothorac Vasc Anesth 2015;29:576-81.

42. Bøtker MT, Vang ML, Grøfte T, et al. Implementing point-of-care ultrasonography of the heart and lungs in an anesthesia department. Acta Anaesthesiol Scand 2017;61:156-65.

43. Jensen MB, Sloth E, Larsen KM, et al. Transthoracic echocardiography for cardiopulmonary monitoring in intensive care. Eur J Anaesthesiol 2004;21:700-7.

44. Moore CL, Copel JA. Point-of-care ultrasonography. N Engl J Med 2011;364:749-57.

45. Rudolph SS, Sørensen MK, Svane C, et al. Effect of prehospital ultrasound on clinical outcomes of non-trauma patients--a systematic review. Resuscitation 2014;85:21-30.

46. Zanobetti M, Scorpiniti M, Gigli C, et al. Point-of-Care Ultrasonography for evaluation of acute dyspnea in the ED. Chest 2017;151:1295-301.

47. Harrell FE, Lee KL, Matchar DB, et al. Regression models for prognostic prediction: advantages, problems, and suggested solutions. Cancer Treat Rep 1985;69:1071-7.

48. Concato J, Feinstein AR, Holford TR. The risk of determining risk with multivariable models. Ann Intern Med 1993;118:201-10.

49. Sasse SA, Chen PA, Berry RB, et al. Variability of cardiac output over time in medical intensive care unit patients. Crit Care Med 1994;22:225-32.

50. Jozwiak M, Monnet X, Teboul JL. Monitoring: from cardiac output monitoring to echocardiography. Curr Opin Crit Care 2015;21:395-401.

51. Shah MR, Hasselblad V, Stevenson LW, et al. Impact of the pulmonary artery catheter in critically ill patients: meta-analysis of randomized clinical trials. JAMA 2005;294:1664-70.

52. Rajaram SS, Desai NK, Kalra A, et al. Pulmonary artery catheters for adult patients in intensive care. Cochrane Database Syst Rev 2013;2:CD003408.

53. Koster G, van der Horst ICC. Critical care ultrasonography in circulatory shock. Curr Opin Crit Care 2017;23:326-33.

54. Lichtenstein DA, Mezière GA. Relevance of lung ultrasound in the diagnosis of acute respiratory failure: the BLUE protocol. Chest 2008;134:117-25.

55. Opdam HI, Wan L, Bellomo R. A pilot assessment of the FloTrac cardiac output monitoring system. Intensive Care Med 2007;33:344-9. 